\title{
Design of Online Washing Process for an Engine Based on Washing Experiments
}

\author{
Zhang Yong ${ }^{1}$, Wu Heng ${ }^{1, a}$, Li Benwei ${ }^{1}$ and Zhao Shufan ${ }^{1}$ \\ ${ }^{1}$ Department of Airborne Vehicle Engineering, Naval Aeronautical and Astronautical University, Yantai Shandong \\ 264001, China
}

\begin{abstract}
In order to develop online washing process of a carrier-based aircraft engine and improve washing efficiency, washing equipments were employed in cold operation state to carry out experimental study on structural strength of spray frame, selection of nozzle and determination of injection parameters of online washing spray system, determination of washing injection timing, determination of drying procedure, liquid-washing experiments of different concentrations and water-washing experiments and so on. The results show that if engine is washed in cold operation state, mid-nozzle with upward offset angel of $7^{\circ} 30^{\prime}$ can be selected and the feed liquid pressure provided by washing vehicle is $0.9 \mathrm{Mpa}$. The start time is 20 s after an engine cold start-up, and sustains 30 s for once washing. After a liquid-washing with the use of washing fluid, the liquid concentration of which is no more than $20 \%$, twice water-washing can be carried out to rinse the almost all cleaning agent remaining in the engine runner(the residue is less than $1 \%$ ). The engine can be thoroughly dried by power-on operation, the procedure of which is engine operating $2 \mathrm{~min}$ in ideal state, then $2 \mathrm{~min}$ in state of $\mathrm{n} 2=85 \%$ and $2 \mathrm{~min}$ of ideal state lastly. Washing will not affect the engine function.
\end{abstract}

\section{Introduction}

As a carrier-based power plant, there are two main purposes of a type of engine in situ washing: one is, considering the sea flight conditions and environment, anti-corrosion washing of engine leaves is carried out to remove the salt spray of the fan, high pressure compressor and other components to prevent corrosion of mechanical parts. The other is, when flow capacity and efficiency of components declined due to fouling and other factors of engine airflow channel resulting in reduction of engine thrust and increase of fuel consumption, the engine airflow channel washing is carried out depending on the situation or combined with the timing of inspection. The main technical contents of engine washing include: special washing agent, washing equipment, washing implementation specifications and washing efficiency assessment [1]. After the development of engine washing equipment and the usage of special washing agent, designing the washing process and developing a washing implementation specification are essential to improve washing efficiency and increase aircraft attendance, as well as provide a basis for engine maintenance procedures.

At present, the United States and Europe and other advanced aviation technology countries have a wide range of research on gas turbine, aero engine washing technology. It mainly includes:

\footnotetext{
${ }^{a}$ Corresponding author : wuheng89810@sina.com
} 
compressor corrosion and blade washing [2-3], modelling and optimization of multiphase flow in online washing [4] as well as the analysis of the effect of washing on engine performance [5-6] and so on. Domestic research on washing technology has just started and mainly focuses on shore-based aero engines. Major studies include: washing technologies [7-9] as well as simulation analysis of washing effectiveness [10-11] and so on. As we can see, washing technology-related researches are carried out at home and abroad. But it could not find related literature about the establishment of engine online washing process based on a large number of washing experiments.

In this paper, cooperated with industrial sector, a large number of washing experiments are designed to carry out based on previous research, it mainly includes experimental study on structural strength of spray frame, selection of nozzle and determination of injection parameters of online washing spray system, determination of washing injection timing, determination of drying process, liquid-washing experiments of different concentrations and water-washing experiments and so on. Finally, according to the experimental results, a certain type of engine online washing process is determined.

\section{Engine washing experiments in cold operation state}

\subsection{Experimental equipments and preparation work}

As the washing parameters in the cold operation state can be adjusted, the washing nozzle flow is small, and the control of corresponding particle size is relatively easy, therefore, cold run immersion and flushing and hot test drying are adopted as an online washing mode by a certain type of carrierbased engine. The test engine used for research on technical state after overhaul qualified is removed from a certain type of carrier-based aircraft. A mechanical valve with relatively high reliability is used by washing car, there are two solution tanks, that is, the same washing solution or rinse solution can be loaded into the solution tank to work together and also the washing solution and pure water can be loaded into the solution tank to work separately. Washing fluid transport power use pneumatic for the system comes with two small high-pressure cylinders. Structural installation diagram is shown in Figure 1. Washing spray frame is shown in Figure 2, finite element is used to obtain the structural static strength and modal analysis of the spray frame and the verification tests of simulated environmental vibration are designed to find that the structural strength of the spray frame could meet the requirements. Cloud diagram of static force calculation (The amount of deformation) and Block diagram of simulated environmental vibration test system are shown in Figure 3 and Figure 4.

A carrier-based aircraft inlet with full state size is adopted and the installation on the gantry simulates the installation status on the airplane. Other experimental equipments mainly include: porcelain probe, high-definition camera, nitrogen bottle (pressure not less than 12MPa), GT-1 washing agent and drinking water.

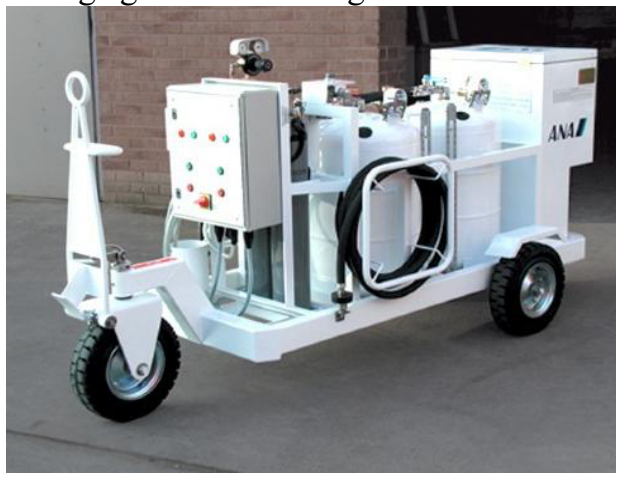

Figure 1. ( $2 \times 120$ litters $)$ Structural appearance of

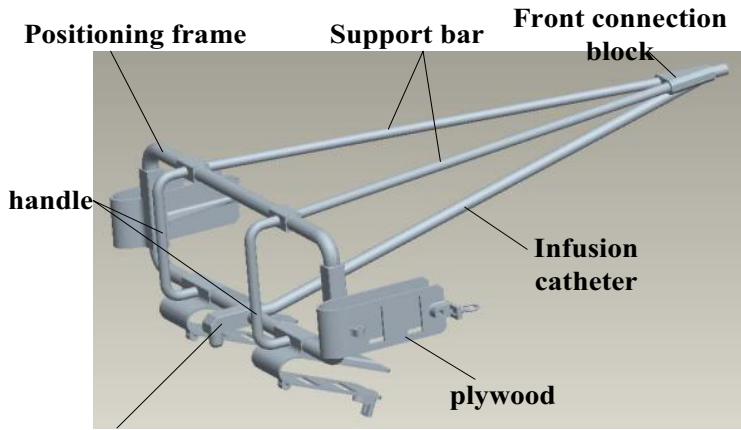

Post-connection block

multi-purpose engine washing equipment

Figure 2. Washing spray frame 


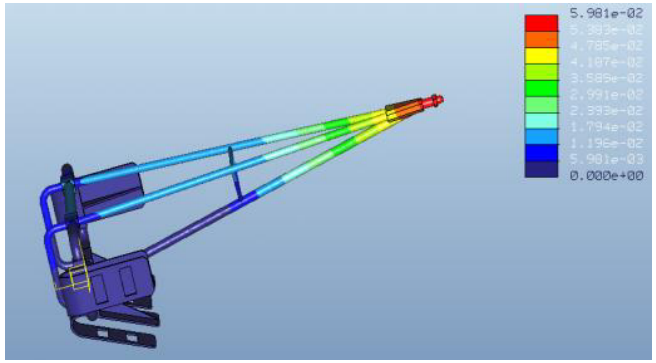

Figure 3. Cloud diagram of static force calculation (The amount of deformation)

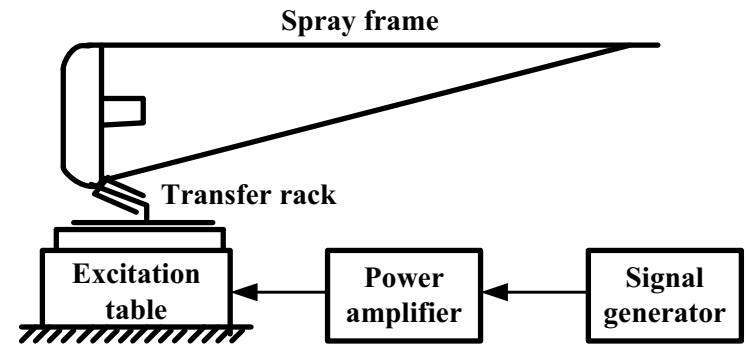

Figure 4. Block diagram of simulated environmental vibration test system

The experiments' preparation mainly includes the following aspects:

(1) install the craft cover only at the outside ducted air-entraining position (aircraft fuel tank pressurization) and seven-stage air-sucking position of high pressure compressor (gas supply from aircraft control system), the remaining interface status is consistent with the installed state. Before the experiments, check the engine function according to the outline requirements of the engine bench washing experiments to ensure that all kinds of parameters and functions are normal;

(2) complete installation of the inlet for test and the transfer section to ensure the same installation state with a carrier-based aircraft inlet;

(3) make sure that the washing vehicle is in good condition;

(4) install the washing spray frame and connect with the washing vehicle, check the connection of the liquid and gas pipeline of the washing vehicle to make sure that it is intact, watertight, no leakage and meets the sealing requirements;

(5) put the monitoring camera in the position aimed at the engine tailpipe to record the engine drain during the whole washing process; Place the camera in front of the test inlet to record water jet during the washing process; record videos and take pictures about the engine water leakage under the engine.

\subsection{Washing experimental methods, process and results}

\subsubsection{Washing nozzle selection and parameter determination}

As nozzle of the injection system is far away from the engine entrance, in order to ensure that the washing fluid will not be sprayed out to inlet wall, the inlet side of engine has a certain water flow coverage and the water flow has the capability of multi-stage fan and compressor cascade penetration, it is necessary to optimize the selection of the numerical range of parameters such as flow rate, pressure and installation angle of the nozzle [12]. Compared with the traditional trial method of determining the engine washing injection parameters, the method of flow field simulation and preselection can greatly reduce the number of tests and also reduce the test cost. Therefore, commercial software FLUENT is used for flow simulation and solution. The droplet size and motion trajectory are taken as the optimization goal, and the droplet on the airflow inlet face of the engine and the penetrating ability are taken as the effect target to carry out numerical simulation by using Linearized Instability Sheet Atomization (LISA) and Spalart-Allmaras ( S-A) models. The specific simulation theory, model and results are described in detail in literature 12. Optimize the selection of the design parameters of nozzle according to a large number of simulation results, that is, when the engine washed in cold operation state, the inlet has certain water flow coverage under the conditions that the spray cone angle is $15 \sim 16^{\circ}$ and the upward offset angel of nozzle is $5 \sim 6^{\circ}$. In addition, the diameter of most atomized droplets of the injection fluid is less than $2 \mathrm{~mm}$, the number of droplets exceeding $2 \mathrm{~mm}$ in diameter is minimal and no more than $3 \mathrm{~mm}$. while engineering practice and research analysis show that: the larger the particle size of the washing droplets formed by the nozzles and the greater the particle speed, the better the washing effect. But oversized and too fast droplets will cause damage to the blade surface, so the appropriate washing fluid particle size is not more than 
$3 \mathrm{~mm}$ (generally, the number of droplets exceeding $2 \mathrm{~mm}$ in diameter should not be too much), the appropriate droplet velocity is close to or slightly larger than the velocity of the flow field. So it concludes that in the range of the spray parameters, the distribution of the spray particle size is reasonable so that it will not cause the fan and compressor blade erosion. The droplet's circumferential and radial distributions are shown in Figure 5, we can see that in this condition, the droplets have the capability of multi-stage fan and compressor cascade penetration so that it is conducive to the realization of engine washing.

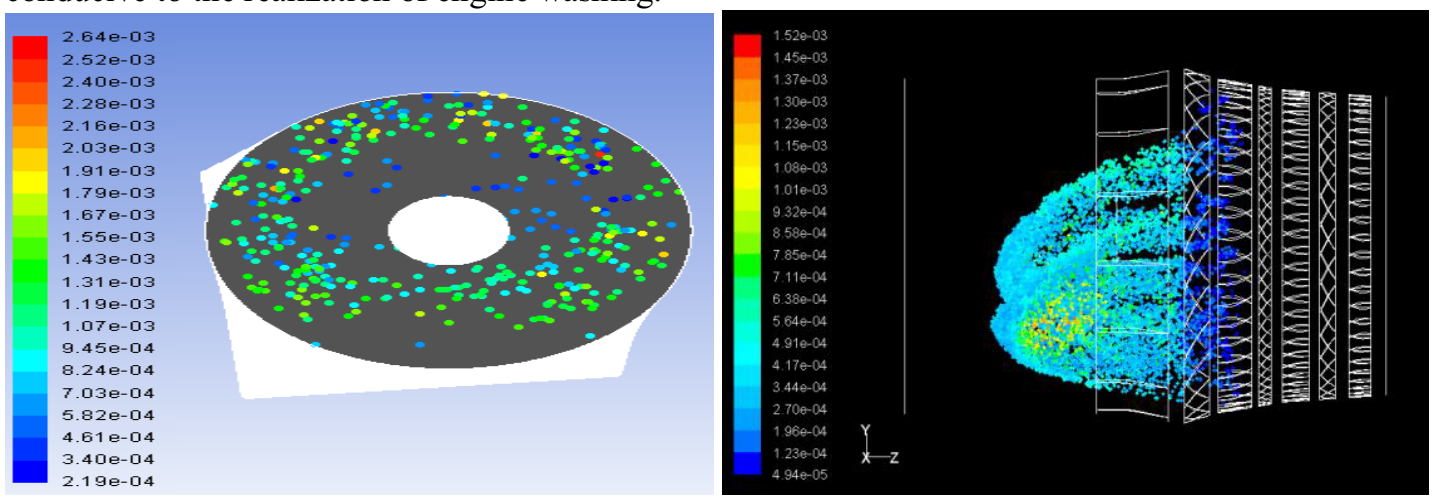

Figure 5. Circumferential and radial distribution of spray droplets

(Cone angle of $16^{\circ}$, upward offset angle of $6^{\circ}$, pressure of $0.8 \mathrm{MPa}$, SMD of 1000)

According to the optimally selected nozzle design parameter data, combined with the nozzle design manual [13], several sets of built-in swirl nozzle are developed. Nozzle test system is adopted in this paper to test the developed nozzle and also make an adjustment of the necessary performance parameters. The schematic diagram is shown in Figure 6. The test system includes several subsystems, such as flow test system in pipe (mainly water flowmeter), optical goniometer, droplet particle size and velocity test system (laser Doppler test system), et al. Among them, the particle test instrument employs PDI provided by Artium of America. The data transform software packet MATLABTM is implanted into information processing system with multi-user data operation and system control functions. The test system achieves the overall measurement of the injection flow field by the automatic control of axes. Through the tests, it is indicated that the nozzles can meet the design requirements. What's more, multi kinds of nozzles and nozzle parameters with adjustment tolerance have been determined, which lays the foundation for further selection of working parameters and nozzle optimization.

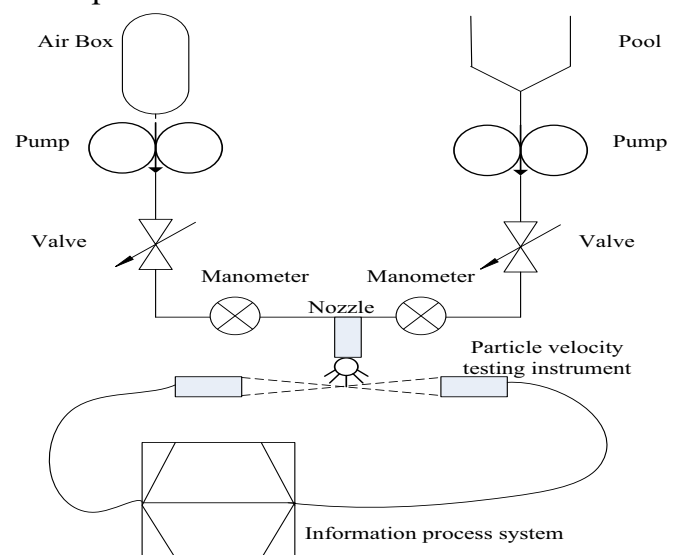

Figure 6. Schematic diagram of experiment system

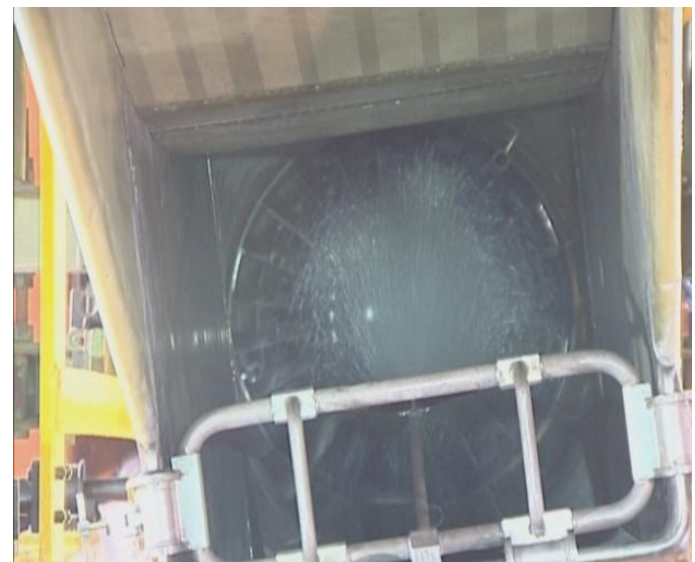

Figure 7. Medium nozzle with upward offset angel of $7^{\circ} 30^{\prime}$

An online washing ground simulation test system of engine flow passage is designed for ensuring the reliable washing under the condition that the inlet and injection equipment are equipped into test 
bench and meanwhile reducing the total test time. Different models of nozzles and their spray parameters are pre-selected in test bench trail. The engine bench tests are carried out according to the results of the pre-selection and verification. The spray effect experiments are carried out in three medium nozzles at different angles under different pressure, respectively there are mid-nozzle with upward offset angel of $8^{\circ} 30^{\prime}$ and the corresponding feed liquid pressure provided by washing vehicle are $0.6 \mathrm{MPa}, 0.7 \mathrm{MPa}, 0.9 \mathrm{Mpa}$ and $1 \mathrm{Mpa}$, mid-nozzle with upward offset angel of $8^{\circ}$ and $7^{\circ}$ $30^{\prime}$ and the corresponding feed liquid pressure provided by washing vehicle is $0.9 \mathrm{Mpa}$. what's more, The spray effect experiments are carried out in small nozzles at different angles under different pressure, respectively there are nozzle with upward offset angel of $8^{\circ} 30^{\prime}, 10^{\circ}$ and $4^{\circ}$ and the corresponding feed liquid pressure provided by washing vehicle is $1 \mathrm{Mpa}$. According to the visual water injection situation from the front of inlet and the transition section near the observation window, it is found that the forward impact force of small nozzle water flow is weak so that the intake air flow is not sufficient to draw the water at high speed into the engine, therefore, there is no need to do the spray effect validation tests under the higher water pressure of the small nozzle in cold operation state. When the two kinds of nozzles washed in cold operation state, check the engine flow passage through the hole detector, it is found that there are water traces in low pressure and high pressure compressor blades at all levels, there is a small amount of water remaining in the test inlet and engine connections, there is more water discharged from the combustion chamber leakage port, and there are a large number of water discharged from the outer duct leakage port, what's more, there is no difference of the remaining water leakage points basically. In view of the combustion chamber leakage port and the outer duct leakage port have a leaky oil pipeline connected to the engine compartment outside so that washing will not affect the engine compartment and aircraft system.

The nozzle installation angle is adjusted to ensure the nozzle around the center, observe the water flow from the front of inlet to find that: mid-nozzle with upward offset angel of $7^{\circ} 30^{\prime}$ can be selected and the feed liquid pressure provided by washing vehicle is $0.9 \mathrm{Mpa}$. It can meet the requirements of the injection range at the engine intake box, the ejection effect is shown in Figure 7.

\subsubsection{Determination of washing injection timing}

The timing of washing injection is determined by reference to the requirements of a certain type of engine channel washing instructions in the outline of the engine bench washing experiments, that is, 10 s after an engine cold start-up, and sustains 30s for spraying. During the experiments, it is found that the engine remained at a high speed after stopping the injection. Check the $\mathrm{n} 2$ speed of multiple engines in the factory in cold operation state to find that the high pressure speed can reach about $20 \%$ after 20 s of the engine's start-up, and also drop to around $20 \%$ after 50 s of the engine's start-up. To ensure that the engine has a high speed when the outfield washing began to spray, the washing injection timing is adjusted and four repetitive verification tests are performed to find that the washing injection timing in cold operation state is finally determined as: 20 s after an engine's cold start-up, and sustains 30 s for spraying.

\subsubsection{Determination of engine drying procedure}

Respectively, dry the engine according to the two kinds of drying procedure developed in the outline of the engine bench washing experiments, eight repetitive verification tests are performed. According to the first drying procedure, that is, dry the engine after engine operating at idle state for $5 \mathrm{~min}$, a total of three trials are conducted to find that after drying the engine piping is waterless, but there is still a small amount of water remaining in the connection between the inlet and engine; according to the second drying procedure, that is, engine operates $2 \mathrm{~min}$ in ideal state, then $2 \mathrm{~min}$ in state of $\mathrm{n} 2=85 \%$ and $2 \mathrm{~min}$ of ideal state lastly. A total of five trials are conducted to find that the engine piping and the connection between the inlet and engine is waterless after drying, that is to say, when the engine operates in state of $\mathrm{n} 2=85 \%$, the residual water in the connection between the inlet and engine will 
be sucked into the engine. Before and after drying, the oil sample is obtained and tested for a total of 13 times to find that there is no moisture content in the oil, and also the oil viscosity, acid value, flash point and other physical and chemical indicators are in line with the requirements.

In summary, to ensure that the residual water in the connection between the inlet and engine can be sucked into the engine when the engine washed in the outfield, the drying procedure which is engine operating $2 \mathrm{~min}$ in ideal state, then $2 \mathrm{~min}$ in state of $\mathrm{n} 2=85 \%$ and $2 \mathrm{~min}$ of ideal state lastly is developed. Two trials are conducted to find that the engine piping is waterless after drying. According to the test result of the seven-stage air-sucking cover between the engine and airplane after each washing and drying, it is found that there are varying degrees of water residue so that when the engine washed, disconnect the aircraft control system and tank pressurized air-entraining piping with the engine, and install the craft cover in the corresponding location.

\subsubsection{Cooperative experiments of liquid washing and water washing}

After determination of the technical state of the engine washing jet system in cold operation state, the washing injection timing and the post-washing drying procedure, washing experiments of three washing fluid ratio $5 \%, 10 \%$ and $20 \%$ are carried out respectively, after spraying washing fluid to the engine airflow channel for $30 \mathrm{~s}$ at the first time, another four times drink water washing are implemented in cold operation state, and then pick up water samples from outer duct leakage port and analyze residual amount of the washing agent in the water sample after each water rinse. Dry the engine after washing, and analyze the obtained oil samples before and after drying. Liquid washing experiment of $10 \%$ concentration in cold operation state is adopted as an example to illustrate the specific experimental process, the experimental process of $5 \%$ and $20 \%$ concentration liquid washing are basically the same.

The specific experimental process of $10 \%$ concentration liquid washing in cold operation state: there is a small amount of washing solution remaining in the connection between the inlet and engine after spraying washing solution for 30 s in cold operation state, as is shown in Figure 8; At the first water washing, there is still a bubble leak in the connection between the boost converter valve and the cited trachea road and there are more bubbles in the water sample obtained from outer duct leakage port; At the second water washing, there is no visible bubble in the water outflowed at the connection between the boost converter valve and the cited trachea road and the same as the water sample obtained from outer duct leakage port; At the third and fourth water washing, there is no bubble in the water sample at all. The first and fourth water samples are shown in Figure 9. Dry the engine after washing, check the airtight piping and the test inlet, there is no water residue.

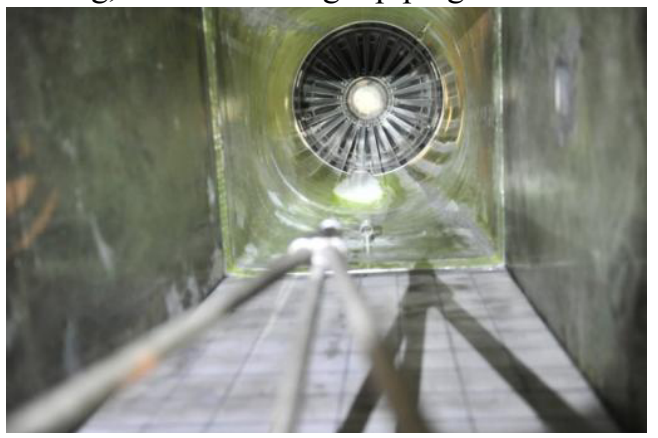

Figure 8. The residue washing fluid in transfer section of inlet and engine junction

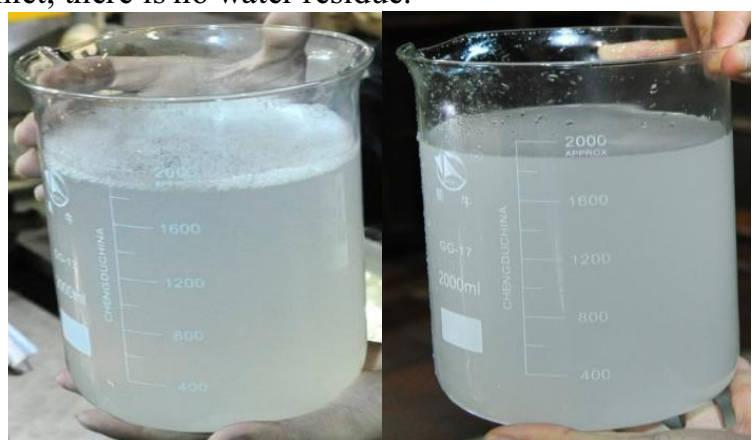

Figure 9. The first and fourth water sample

According to the test results of water sample after each water washing, it is found that after a $10 \%$ concentration liquid washing, twice water washing can be carried out to rinse the almost all cleaning agent remaining in the engine runner, and also after a $5 \%$ concentration liquid washing, twice water washing can be carried out, in this condition the amount of washing agent residue has been very low. Due to the timing of getting water sample is not exactly the same, it is likely that the amount of 
washing agent residue of a $10 \%$ concentration liquid washing and twice water washing is less than that of a 5\% concentration liquid washing and twice water washing. But according to the test results, it is still concluded that after a different concentration liquid washing, twice water washing can be carried out to rinse the almost all cleaning agent remaining in the engine runner (the residue is less than $1 \%$ ). The schematic diagram of residual amount of washing fluid in water sample after a different number of water rinses is shown in Figure 10.

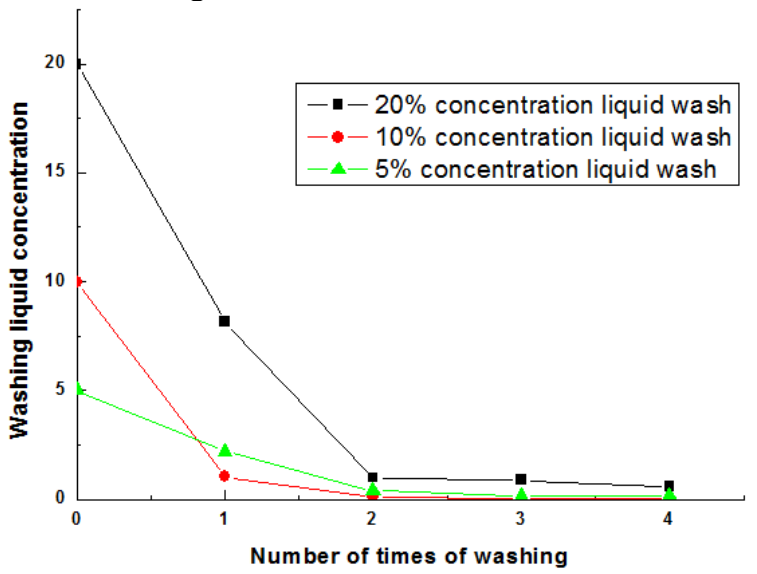

Figure 10. Schematic diagram of residual amount of washing fluid in water sample after a different number of water rinse

After a $20 \%$ concentration liquid washing and four times drink water washing, another four times drink water washing are carried out in cold operation state without drying. After a total of nine times washing in cold operation state, dry the engine according to the drying procedure of outfield engine washing, That is, engine runs for $2 \mathrm{~min}$ in ideal state, then $2 \mathrm{~min}$ in state of $\mathrm{n} 2=85 \%$ and $2 \mathrm{~min}$ of ideal state lastly, after drying, check the airtight piping and the test inlet, there is no water residue.

In summary, according to the results of engine washing experiments with three different concentrations of washing fluid and the test results of the residual amount of the washing agent in the water sample after each water rinse, it is found that after a liquid-washing with the use of washing fluid, the liquid concentration of which is no more than $20 \%$, twice water-washing can be carried out to rinse the almost all cleaning agent remaining in the engine runner (the residue is less than 1\%).

\section{Development of the washing process based on experimental results}

According to the results of liquid-washing experiments of three different concentrations and waterwashing experiments, the washing process in cold operation state can be determined as follows:

(1) disconnect the aircraft control system and tank pressurized air-entraining piping with the engine, and install the craft cover in the corresponding location;

(2) 20 s after an engine cold start-up, and sustains 30 s for spraying the washing solution;

(3) 20s after an engine cold start-up, and sustains 30s for spraying the drinking water;

(4) after twice water-washing, the engine can be thoroughly dried by power-on operation, the procedure of which is engine operating $2 \mathrm{~min}$ in ideal state, then $2 \mathrm{~min}$ in state of $\mathrm{n} 2=85 \%$ and $2 \mathrm{~min}$ of ideal state lastly;

(5) restore connection of the aircraft control system and tank pressurized air-entraining piping with the engine.

After the engine washing is finished, check the engine function according to the outline requirements of the engine bench washing experiments, it is find that all kinds of parameters change reasonably and the engine is functioning properly, that is to say, Washing will not affect the normal operation of the engine. 


\section{Conclusions}

Through the study of this paper, the following conclusions are drawn:

(1) through the engine bench washing experiments, it is possible to determine the technical state of the engine washing jet system, the washing injection timing and the post-washing drying procedure, that is, if engine is washed in cold operation state, mid-nozzle with upward offset angel of $7^{\circ} 30^{\prime}$ can be selected and the feed liquid pressure provided by washing vehicle is $0.9 \mathrm{Mpa}$. The start time is 20 s after an engine cold start-up, and sustains 30s for once washing. The engine can be thoroughly dried by power-on operation, the procedure of which is engine operating $2 \mathrm{~min}$ in ideal state, then $2 \mathrm{~min}$ in state of $\mathrm{n} 2=85 \%$ and $2 \mathrm{~min}$ of ideal state lastly.

(2) according to the results of engine washing experiments with three different concentrations of washing fluid and the test results of the residual amount of the washing agent in the water sample after each water rinse, it is found that after a liquid-washing with the use of washing fluid, the liquid concentration of which is no more than $20 \%$, twice water-washing can be carried out to rinse the almost all cleaning agent remaining in the engine runner(the residue is less than $1 \%$ ). Washing will not affect the engine function.

\section{References}

1. E Syverud, L. E. Bakken, Online Water Wash Tests of GE J85-13, JOT, 129: 136-142(2007).

2. R Kurz, K Brun, Fouling Mechanisms in Axial Compressors, JOEFGTAP, 134(3): 1-9(2012).

3. F. C. Mund, P. Pilidis, Online Compressor Washing: A Numerical Survey of Influencing Parameters, POTIOME, PA: JOPAE, 219: 13-21(2005).

4. E. M. Wahba, H. Nawar, Multiphase Flow Modeling and Optimization for Online Wash Systems of Gas Turbines, AMM, 37: 7549-7560(2013).

5. E. A. Ogbonnaya, Gas Turbine Performance Optimization Using Compressor Online Water Washing Technique, E, 3: 500-507(2011).

6. S Demircioglu, Detail Analysis of Compressor Online Wash Impact in the Gas Turbine Performance(Master thesis, Germany: FH Offenburg, 2008).

7. B. W. Li, X. X. Wang, G. C. Hu, Research on cleaning technology of turbojet engine, A, 36(1): 12-16(2000).

8. C. B. Yuan, H. Z. Miao, X. Huang, Washing Technology of Turbo-shaft Engine for Military Helicopter, AMAE, 5: 36-38(2010).

9. Y. Y. Yang, Y. Zhao, Influence analysis of a certain engine shipboard washing schedule of based on environment test, JONAAAU, 29(2): 168-172(2014).

10. S. W. Chen, C. Zhang, H. Shi, Numerical Study on the Impact of Fouling on Axial Compressor Stages, JOPT, 33(3): 377-383(2012).

11. C. H. Wang, Z. P. Xu, X. Y. Yang, Numerical simulation study on two phase flow field of cleaning aero engine, SST, 11(2):101-105(2015).

12. K. Y. Jiang, W. Shen, B. W. Li, Parameters optimizing for a turbofan engine cleaning system using flow field simulation, JOAP, 26(3):481-488(2011).

13. L. Y. Hou, X. C. Hou, Technical manual of nozzle(China Petrochemical Press, Beijing, 2002). 